\title{
Comparison of Congenital Tooth Deficiencies Seen in Permanent Teeth in Individuals with Unilateral Cleft Lip and Palate to Those without Cleft Lip and Palate
}

\author{
Ege Dogan \\ Orthodontists, Private Office, Izmir, Turkey \\ Email: dtegedogan@hotmail.com
}

How to cite this paper: Dogan, E. (2021) Comparison of Congenital Tooth Deficiencies Seen in Permanent Teeth in Individuals with Unilateral Cleft Lip and Palate to Those without Cleft Lip and Palate. Open Journal of Stomatology, 11, 188-196. https://doi.org/10.4236/ojst.2021.115016

Received: April 5, 2021

Accepted: May 16, 2021

Published: May 19, 2021

Copyright ( 2021 by author(s) and Scientific Research Publishing Inc. This work is licensed under the Creative Commons Attribution International License (CC BY 4.0).

http://creativecommons.org/licenses/by/4.0/

(c) (i) Open Access

\begin{abstract}
Objective: To compare the congenital tooth deficiencies seen in permanent dentition in individuals with unilateral cleft lip and palate (UCLP) to non-cleft individuals with Angle Class I malocclusion. Method: The study was performed on orthopantomograph films of 50 individuals with UCLP aged between 12 16 years and 50 individuals with Angle Class I malocclusion individuals aged between 13 - 15 years. Individuals with UCLP; 32 clefts were on the left side and 18 clefts were on the right side. Permanent third molar teeth deficiency was excluded from the study. Results: In 50 individuals with UCLP; 35 (70\%) upper lateral incisors were congenitally deficient in the cleft area, while 15 (30\%) missing lateral teeth were found in the non-cleft side. In control group, $12(24 \%)$ of 50 patients had congenital lateral incisor deficiency. Congenital deficiency of upper lateral incisor in UCLP; the cleft area was higher than the non-cleft side $(\mathrm{p}<0.01)$. The difference was statistically important when compared with the control group ( $\mathrm{p}<0.001)$. In 50 individuals with UCLP; while $27(54 \%)$ of second premolar teeth were congenitally deficient in cleft side, 23 (46\%) were missing in non-cleft side. In control group, 18 (36\%) congenital second premolar deficiency was detected. However, second premolar congenital deficiency was higher in UCLP group when compared to control group ( $\mathrm{p}<0.01$ ). Conclusion: The deficiency of the lateral incisors in the cleft side is more often congenitally deficient than upper second premolar teeth and this should be considered in the treatment planning from an early age.
\end{abstract}

\section{Keywords}

Congenital Teeth Deficiency, Unilateral Cleft Lip and Palate 


\section{Introduction}

Cleft lip and palate (CLP) is a congenital development anomaly, and we encounter the loss of substance caused by cleft in the upper jaw. The cleft lip and/or palate constitute approximately $65 \%$ of all congenital craniofacial anomalies. The prevalence of this congenital anomaly in the world was $1 / 700$ - 1000 ratio, this ratio is seen as $1 / 800$ in Turkey. The anomaly is the most common (1:500) in those of Asian origin and moderately frequent in the white race (1:750) and the rarest (1:1000 or less) in the black race. When speech and sometimes hearing problems are added to the difference in appearance, the child appears with great psychological problems. Occlusion disorders and dental anomalies increase with the severity of the cleft. Treatment of CLP requires a multidisciplinary approach. Plastic surgeon, orthodontist and speech therapist, especially pediatrician, geneticist, ENT doctor, child psychiatrist, pedodontist, dentist are the most important elements of this team [1] [2] [3] [4] [5].

Dental anomalies are frequently seen in individuals with CLP. The severity of the cleft also determines the direction and size of the development of teeth and occlusion. Generally, both deciduous and permanent dentitions are affected, but in permanent dentition, these anomalies occur at a higher rate [5]-[12].

General population has a prevalence of $0.027 \%$ to $10.1 \%$ in congenitally missing teeth. This varies according to race and geographic location [8] [9]. Some authors reported that in general population, the most commonly missing teeth are the maxillary lateral incisors, while others reported that it is the mandibular second premolars with $3.4 \%$ frequency and this is followed by the maxillary lateral incisors with 2.2\% frequency [2] [4] [9] [12] [13] [14] [15].

In children with CLP, in both deciduous and permanent dentition, upper lateral incisors were reported to be the most commonly missing teeth in the cleft region and the upper second premolars were reported to be more frequently deficient than the normal population. Congenitally absent teeth were seen more unilaterally than the bilaterally, but in second premolar teeth; the bilateral absence was found 1.5 times more often than unilateral absence [7] [12] [13] [16] [17]. It has been reported that congenital tooth deficiency is between $1.5 \%$ and $6.6 \%$ in the general population, and congenital deficiency of the lateral teeth is less common in the normal population than the deficiency of the second premolar teeth [1] [4] [5] [7] [11] [14].

Ranta [1] reported that the most commonly missing teeth in the cleft area is the maxillary lateral incisor which is followed by the maxillary second premolars. This is explained by the proximity of the cleft in the lateral incisor region.

$\mathrm{Wu}$ et al. [10], severity of the cleft increases the prevalence of lateral incisor agenesis. Dental agenesis is seen mostly in left side regardless of the cleft side. This could be explained by overlapping etiopathogenetic factors where dental agenesis and clefts are involved.

Shapira et al. [4] [5], found that maxillary lateral incisors and second premolars are not seen more frequently on the left side, which also had a higher fre- 
quency of clefting. In their study, more missing teeth were seen in non-cleft side in unilateral cleft and alveolus patients. Baek and Kim [8] also found that lower prevalence of hypodontia in non-cleft side. Jamilian et al. [9] found out that there is no difference in regard to the gender of the patients, the left side is affected more and the missing upper lateral incisor is seen more in the cleft side than the non-cleft side.

Individuals with CLP present significantly more dental anomalies, even outside the cleft area, than do individuals without clefts in different prevalence.

The aim of this study is to compare the congenital tooth deficiencies seen in permanent teeth in patients in Aegean region with UCLP with individuals without CLP in Turkey. Therefore, the null hypothesis of this study was that tooth agenesis and dental structural anomalies are not different between the various types of UCLP.

\section{Material and Method}

This retrospective study was done on ortopantomograph films taken before treatment from 100 patients who applied for orthodontic treatment. 50 individuals with UCLP between the ages of 12 - 16 years were compared with the 50 individuals with Angle Class I malocclusion with no CLP aged between 13 - 15 years.

In the patients with UCLP; 32 of them have cleft on the left side while 18 of them have cleft on the right side. The congenitally deficient teeth were recorded from the ortopantomograph films. In this study, third molars were not included in the evaluation.

The inclusion criteria were as follows:

- Patients with UCLP aged between 12 - 16 years and patients with Angle Class I malocclusion with no CLP aged between 12 - 16 years.

- Patients had no syndromes, no extraction of permanent teeth, no endodontic/prosthodontic/orthodontic treatment, and no trauma to any tooth before the initiation of orthodontic treatment.

- The ortopantomograph films taken before treatment from the patients showed any artifacts.

The exclusion criteria were as follows:

- Patients with UCCLP not aged between 12 - 16 years and patients with Angle Class I malocclusion with no CLP not aged between 13 - 15 years.

- Patients had syndromes, extraction of permanent teeth, endodontic/prosthodontic/orthodontic treatment, and trauma to the tooth before the initiation of orthodontic treatment.

- The ortopantomograph films taken before treatment from the patients showed artifacts.

\section{Statistical Analysis}

The number of the patients to be included in the study was determined by Power analysis carried out by using the G Power analysis software. A level of 0.05 was 
accepted as statistically significant. Ortopantomograph films were reconducted on randomly selected radiographs of 25 patients after an interval of 1 month and intra-observer reliability was evaluated using intraclass correlation coefficients (ICC). All statistical analyses were performed by SPSS version 22. A paired sample t-test was used for the intra-group comparisons and an independent sample t-test was used for the inter-group comparisons.

\section{Results}

The intra-class correlation coefficients (ICCs) values were above 0.938 , thus confirming reliability. The power analysis showed that 41 patients were required for each group. To increase the power of the study, it was decided to include $50 \mathrm{pa}$ tients for each group to achieve a representation rate of over $95 \%$.

In patients with UCLP; there is congenital lateral incisor deficiency at the rate of $70 \%$ in the cleft region, $30 \%$ on the non-cleft side and $24 \%$ in the control group (Figure 1). Patients with UCLP compared to the control group; congenital deficiency of upper lateral incisor was seen more in the cleft group $(\mathrm{p}<0.001)$ (Figure 2). Congenital deficiency of the lateral incisor in patients with UCLP; It was found more in the cleft region than the non-cleft side $(p<0.01)$ (Figure 3$)$.

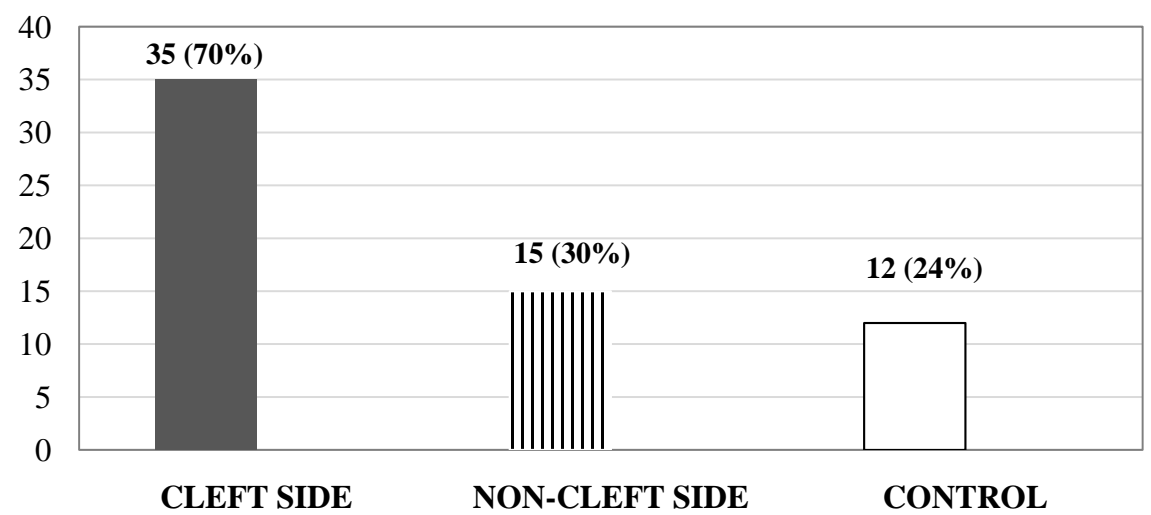

Figure 1. Congenital deficiency of upper lateral incisor in UCLP (cleft side/non-cleft side) and Control group.

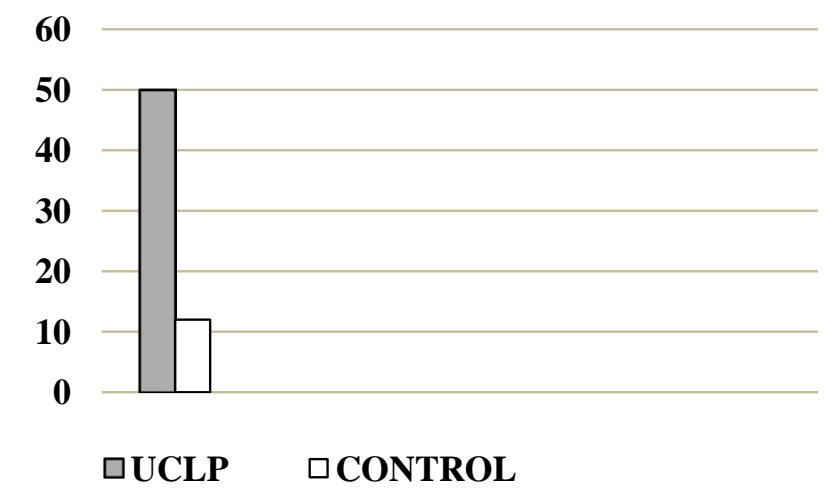

Figure 2. Congenital deficiency of upper lateral incisor in UCLP and Control group ( $\mathrm{p}<$ $0.001)$. 
In patients with UCLP; $54 \%$ in the cleft side, $46 \%$ in the non-cleft side, $36 \%$ in the control group, second premolar tooth congenital deficiency was detected (Figure 4). Patients with UCLP compared to the control group second premolar congenital deficiency was seen more in the cleft group ( $\mathrm{p}<0.01$ ) (Figure 5). Congenital deficiency of the upper second premolar tooth in patients with UCLP; is not statistically different in the cleft side than the non-cleft side $(p>0.05)$ (Figure 6).

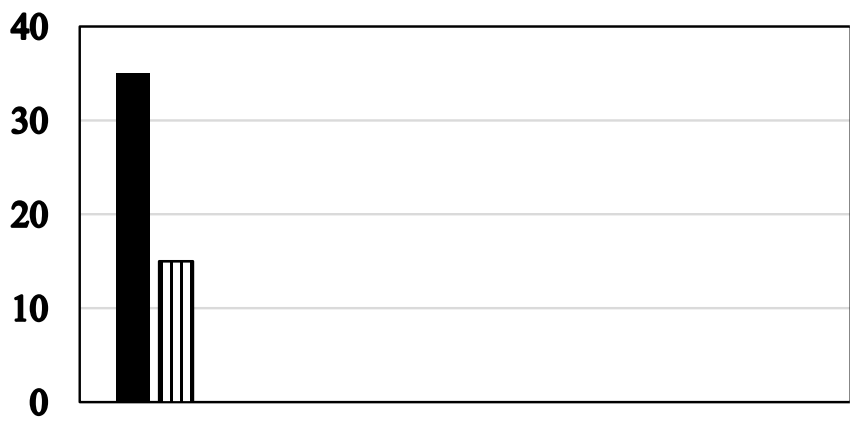

- CLEFT SIDE $\square$ NON-CLEFT SIDE

Figure 3. Congenital deficiency of upper lateral incisor of UCLP in cleft side and in non-cleft side $(\mathrm{p}<0.01)$.

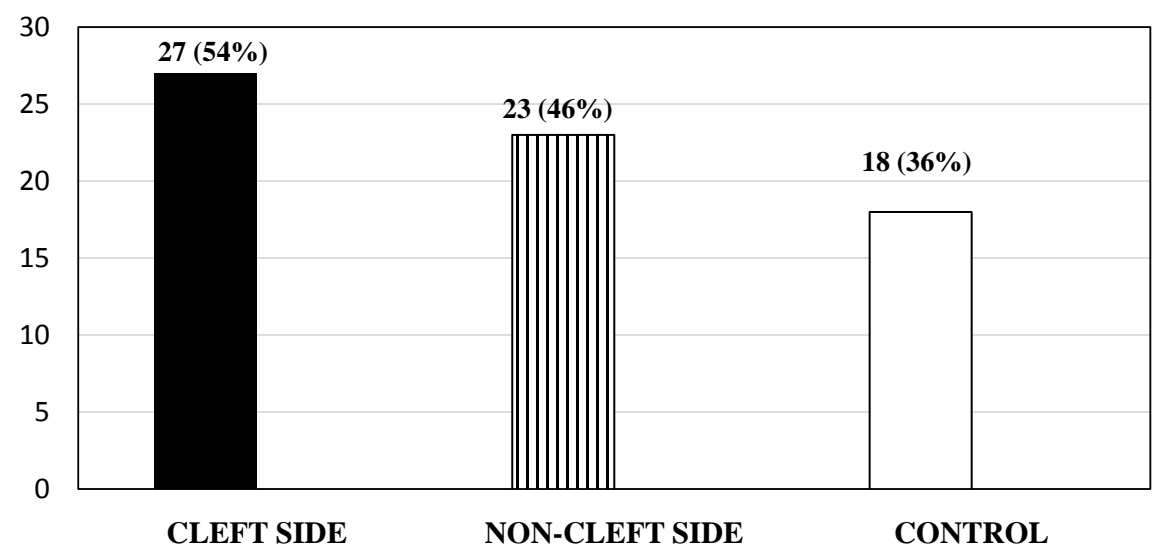

Figure 4. Congenital deficiency of upper second premolar in UCLP (cleft side/non-cleft side) and Control group.

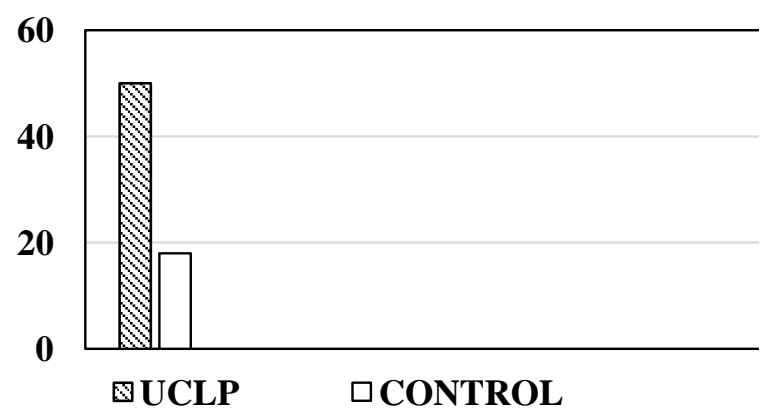

Figure 5. Congenital deficiency of upper second premolar in UCLP and control group (p $<0.01)$. 


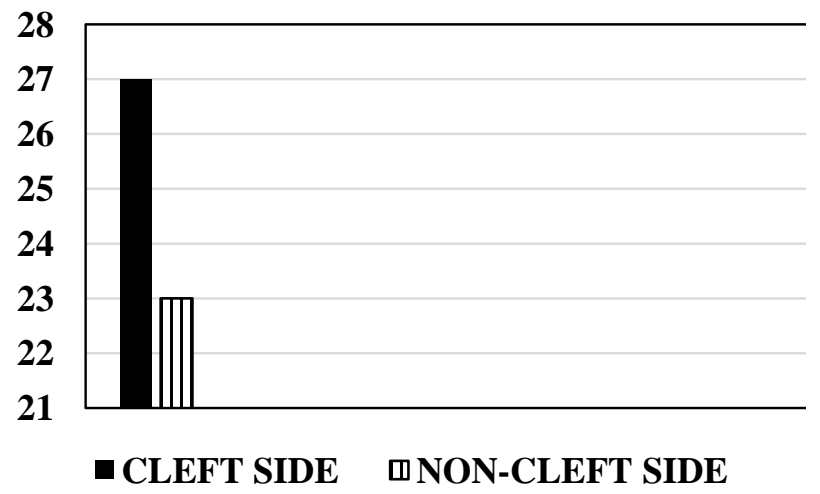

Figure 6. Congenital deficiency of upper second premolar of UCLP in cleft side and in non-cleft side $(\mathrm{p}>0.05)$.

\section{Discussion}

The number of missing teeth has a great role in planning and managing the orthodontic treatment. In CLP patients the number of missing teeth is higher, which causes additional complications in treatment planning. In children with clefts; in both deciduous and permanent dentition, lateral incisors were reported to be the most common missing teeth in the cleft region, and the upper second premolars were reported to be more frequently deficient than the normal population [7] [9] [18].

Congenital deficiency of the lateral tooth in patients with CLP can also lead to anomalies in the canine teeth. Vichi and Franchi [18], examined the eruption of the upper permanent canine teeth in the group of 77 children with CLP. They reported that the canine teeth was positioned mesially in congenital deficiency of the permanent lateral, however, the canines were located distally in the presence of supernumerary teeth.

Rullo et al. [12] found out that in their study that the CLP patients had congenitally missing lateral incisor in $40 \%$ of their samples and $30 \%$ of their samples showed supernumerary teeth at the incisor region.

Shapira et al. [5] found in their study that $74 \%$ of congenitally missing maxillary lateral incisor and \%18 missing second premolar in cleft lip, cleft palate of CLP patients. In CLP patients, hypodontia regardless of the cleft region was found to be higher than the non-cleft patients. The frequency of missing teeth in non-cleft side in CLP patients were $7.5 \%$ to $32.3 \%$ for the maxillary second premolars and $3.1 \%$ to $10.4 \%$ for the maxillary lateral incisors. In unilateral CLP patients, missing teeth were found on the same side with the cleft, which is commonly the left side. In non-cleft patients, missing maxillary incisors and maxillary and mandibular first and second premolars were found more bilaterally than unilaterally.

Shapira Y et al. [4], studied the prevalence of missing teeth in clefts, both in cleft side and non cleft side by using radiographs of 278 patients aged between 5 - 18 year old who had CLP. In the cleft side, the most frequently missing teeth was maxillary permanent lateral incisors (259 teeth) followed by the maxillary 
(47 teeth) and mandibular (23 teeth) second premolars. In the non cleft side, maxillary second premolars ( 12 teeth) were the mostly missing teeth, followed by the maxillary lateral incisors (10 teeth) and mandibular second premolars (6 teeth).

In this study, congenital lateral incisor deficiency was seen at the rate of $70 \%$ (35 teeth) in the cleft side, 30\% (15 teeth) in the non-cleft side in UCLP while it was $24 \%$ (12 teeth) in the control group. But, congenital deficiency of second premolar tooth in patients with UCLP was seen $54 \%$ (27 teeth) in the cleft side, $46 \%$ (23 teeth) in the non-cleft side, while it was 36\% (18 teeth) in the control group.

In CLP patients and in their non-effected siblings, tooth ages are seen more than the general population due to the relationship between tooth and cleft formation due to timing of development and anatomical location. In the cleft area, with a prevalence of $56.1 \%$ to $74 \%$, maxillary lateral incisors are the most commonly missing teeth. The prevalence of tooth agenesis outside the cleft region in cleft patients is $27 \%$, with the second premolars being affected most often [7]. By contrast, Bartzela et al. [19] stated that dental problems related with the cleft don't seem to be restricted to the maxilla because of the prevalence of agenesis of lower second premolar was only three times higher than that in the normal population $(2.9 \%-3.2 \%)$. Besides, Konstantonis et al. [20] stated that $50 \%$ of the cleft patients had tooth agenesis and $18 \%$ had microdontia. Tooth agenesis mostly occurred in lateral incisors in maxilla and second premolars in maxilla and mandibula. The significant association between quadrants with tooth agenesis and quadrants with no tooth agenesis indicates that tooth agenesis is not directly related to the disruptive osseous defect occuring at the cleft side but is rather a genetically controlled anomaly related to the orofacial cleft process.

Some of the investigators stated that specific surgical procedures, such as early periosteoplasty or neonatal closure of the hard palate, might influence the increased prevalence of tooth agenesis in CLP patients [2]. Thus, some other studies have stated that surgical interventions don't have an effect on tooth agenesis [10] [12] [16] [19].

Insufficient tissue in the medial nasal and/or maxillary process during embryological development may cause lateral incisors to be absent mesially and/or distally. However, the high prevalence of agenesis outside the cleft area points to a common genetic background for hypodontia and clefts [20] [21].

There are many theories explaining the absence of teeth in cleft patients including genetic and environmental factors. These factors may be responsible for both the formation of the clefts and the hypodontia in affected children. Evaluation of MSX1 gene as the common candidate gene of nonsyndromic congenital hypodontia and CLP is supporting the hypothesis of a possible relationship between CLP and congenitally missing maxillary lateral incisors [19] [22].

\section{Conclusions}

Diagnosis of dental anomalies and dental occlusion is very important in orthodontic treatment planning of patients with CLP. In this study, congenital defi- 
ciency of lateral incisor is seen much more than upper second premolar teeth. It is important that the lateral incisors, especially in the cleft side, are found to be congenitally deficient, and this should be taken into consideration in treatment planning from an early age.

One of the limitations of this current study is the small number of patients which makes it harder to generalize the results. Larger sample size with different ethnicities should be included with multi-center studies to generalize the results.

\section{Conflicts of Interest}

The author declares no conflicts of interest regarding the publication of this paper.

\section{References}

[1] Lourenco Riberio, L., Teixeira Das Neves, L., Costa, B. and Riberio Gomide, M. (2003) Dental Anomalies of the Permanent Lateral Incisors and Prevalence of Hypodontia outside the Cleft Area in Complete Unilateral Cleft Lip and Palate. The Cleft Palate-Craniofacial Journal, 40, 172-175. https://doi.org/10.1597/1545-1569_2003_040_0172_daotpl_2.0.co_2

[2] Ranta, R. (1986) A Review of Tooth Formation in Children with Cleft Lip/Palate. American Journal of Orthodontics and Dentofacial Orthopedics, 90, 11-16. https://doi.org/10.1016/0889-5406(86)90022-3

[3] Ranta, R. and Tulensalo, T. (1988) Symmetry and Combinations of Hypodontia in Non-Cleft and Cleft Palate Children. Scandinavian Journal of Dental Research, 96, 1-8. https://doi.org/10.1111/j.1600-0722.1988.tb01400.x

[4] Shapira, Y., Lubit, E., Kuftinec, M.M. and Stom, D. (2000) Hypodontia in Children with Various Types of Clefts. The Angle Orthodontist, 70, 16-21.

[5] Shapira, Y., Lupit, E., Kuftinec, M.M. and Stom, D. (1999) Congenitally Missing Second Premolars in Cleft Lip and Cleft Palate Children. American Journal of Orthodontics and Dentofacial Orthopedics, 115, 396-400. https://doi.org/10.1016/S0889-5406(99)70258-1

[6] Symons, A.L., Stritzel, F. and Stamation, J. (1993) Anomalies Associated with Hypodontia of the Permanent Lateral Incisor and Second Premolar. Journal of Clinical Pediatric Dentistry, 17, 109-111.

[7] Eerens, K., Vlietinck, R., Heidbuchel, K., Van Olmen, A., Derom, C., Willems, G. and Carels, C. (2001) Hypodontia and Tooth Formation in Groups of Children with Siblings without Cleft, and Nonrelated Control. The Cleft Palate-Craniofacial Journal, 38, 374-378. https://doi.org/10.1597/1545-1569_2001_038_0374_hatfig_2.0.co_2

[8] Baek, S.H. and Kim, N.Y. (2007) Congenital Missing Permanent Teeth in Korean Unilateral Cleft Lip and Alveolus and Unilateral Cleft Lip and Palate Patients. The Angle Orthodontist, 77, 88-93. https://doi.org/10.2319/113005-419R.1

[9] Jamilian, A., Perillo, L. and Rosa, M. (2015) Missing Upper Incisors: A Retrospective Study of Orthodontic Space Closure versus Implant. Progress in Orthodontics, 16, Article No. 2. https://doi.org/10.1186/s40510-015-0072-2

[10] Wu, T.T., Chen, P.K., Lo, L., Cheng, M.C. and Ko, E.W. (2011) The Characteristics and Distribution of Dental Anomalies in Patients with Cleft. Chang Gung Medical Journal, 34, 306-314. 
[11] Jamilian, A., Lucchese, A., Darnahal, A., Kamali, Z. and Perillo, L. (2016) Cleft Sidedness and Congenitally Missing Teeth in Patients with Cleft Lip and Palate Patients. Progress in Orthodontics, 17, 14. https://doi.org/10.1186/s40510-016-0127-z

[12] Rullo, R., Festa, V.M., Rullo, R., Addabbo, F., Chiodini, P., Vitale, M., et al. (2015) Prevalence of Dental Anomalies in Children with Cleft Lip and Unilateral and Bilateral Cleft Lip and Palate. European Journal of Paediatric Dentistry, 16, 229-232.

[13] Wada, T. and Miyazaki, T. (1975) Growth and Changes in Maxillary Arch Form in Complete Unilateral Cleft Lip and Palate Children. The Cleft Palate-Craniofacial Journal, 12, 115-130.

[14] Polder, B.J., Vanot Hof, M.A., Van der Linden, F.P. and Kuijpers-Jagtman, A.M. (2004) A Meta-Analysis of the Prevalence of Dental Agenesis of Permanent Teeth. Community Dentistry and Oral Epidemiology, 32, 217-226.

https://doi.org/10.1111/j.1600-0528.2004.00158.x

[15] Tortora, C., Meazzini, M.C., Garattini, G. and Brusati, R. (2008) Prevalence of Abnormalities in Dental Structure, Position, and Eruption Pattern in a Population of Unilateral and Bilateral Cleft Lip and Palate Patients. The Cleft Palate-Craniofacial Journal, 45, 154-162. https://doi.org/10.1597/06-218.1

[16] Lekkas, C., Latief, B.S., ter Rahe, S.P. and Kuijpers-Jagtman, A.M. (2000) The Adult Unoperated Cleft Patient: Absence of Maxillary Teeth outside the Cleft Area. The Cleft Palate-Craniofacial Journal, 37, 17-20. https://doi.org/10.1597/1545-1569(2000)037<0017:TAUCPA >2.3.CO;2

[17] Vieira, A.R., McHenry, T.G., Daack-Hirsch, S., Murray, J.C. and Marazita, M.L. (2008) Candidate Gene/Loci Studies in Cleft Lip/Palate and Dental Anomalies Finds Novel Susceptibility Genes for Clefts. Genetics in Medicine, 10, 668-674. https://doi.org/10.1097/GIM.0b013e3181833793

[18] Vichi, M. and Franchi, L. (1996) Eruption Anomalies of the Maxillary Permanent Cuspids in Children with Cleft Lip and/or Palate. Journal of Clinical Pediatric Dentistry, 20, 149-153.

[19] Bartzela, T.N., Carels, C.E.L., Bronkhorst, E.M., Rønning, E., Rizell, S. and Kuijpers-Jagtman, A.M. (2010) Tooth Agenesis Patterns in Bilateral Cleft Lip and Palate. European Journal of Oral Sciences, 118, 47-52. https://doi.org/10.1111/j.1600-0722.2009.00698.x

[20] Konstantonis, D., Alexandropoulos, A., Konstantoni, N. and Nassika, M. (2017) A Cross-Sectional Analysis of the Prevalence of Tooth Agenesis and Structural Dental Anomalies in Association with Cleft Type in Non-Syndromic Oral Cleft Patients. Progress in Orthodontics, 18, 20. https://doi.org/10.1186/s40510-017-0169-x

[21] Vieira, A.R. (2003) Oral Clefts and Syndromic Forms of Tooth Agenesis as Models for Genetics of Isolated Tooth Agenesis. Journal of Dental Research, 82, 162-165. https://doi.org/10.1177/154405910308200303

[22] Hovorakova, M., Lesot, H., Peterkova, R. and Peterka, M. (2006) Origin of the Deciduous Upper Lateral Incisor and Its Clinical Aspects. Journal of Dental Research, 85, 167-171. https://doi.org/10.1177/154405910608500210 